\title{
Amérique sans nom
}

[post-scriptum pour un quincentenaire]

\section{Jean Morisset}

Université du Québec à Montréal

\begin{abstract}
«ll y avait une surprise qui, malgré notre appétit, nous fit presque oublier le dîner somptueux qu'on s'apprêtait à nous servir. Parut à l'improviste une jeune femme qui nous coupa le souffle: un des plus beaux spécimens de brune dont un lieutenant de vaisseau puisse prétendre rêver. Une silhouette ravissante aux lignes pleines et voluptueuses, des traits d'une beauté parfaite, des yeux noirs geais au regard alangui, et la plus luxuriante des chevelures qui aient jamais frisé sur terre. Bref, tout ce qu'il fallait pour conduire une escouade à la poésie ou au suicide.»
\end{abstract}

Traduit de McKenny, United States and Democracy Review jullet 1839, et rapporté par Georges Blond dans Histoire de la Filibuste. Paris: Stock, 1969.

\footnotetext{
Q i je fais appel ici aux filles de la flibuste, de la Rochelle et de la Caraỉbe, pour appareiller vers la souveraineté géographique de l'Amérique; si je fais appel aux filles du grand large et aux filles de la réserve, en guise de post-scriptum à tous les siècles qui nous ont vu naitre, c'est qu'il y a un quincentenaire qui rôde quelque part et risque fort de nous by-passer sans la moindre hésitation.

Au moment où se joue, au Canada, à coup de commissions d'enquêtes et d'impératifs juridiques, l'une des dernières grandes "rondes politiques" prétendant fixer le destin d'une Franco-Amérique toujours restée en marge de son propre avènement, qu'avons-nous à déclarer? Voici:
}

1492-1992: cinq-centième anniversaire de la découverte du nouveau monde!

1492-1992: cinq-centlème anniversaire du génocide fondateur de ses ressortissants aborigènes! 
Et la Franco-Amérique, où se situe-t-elle exactement dans tout cela?

Au moment, donc, où l'Europe s'apprête à se célébrer elle-même à travers nous, qui sommes-nous et que sommes-nous? Qu'avons-nous été au juste et vers quel concordat historique nous dirigeons-nous inéluctablement?

Si la Franco-Amérique se retrouve complètement absente, jusqu'à maintenant, aussi bien des célébrations de la découverte que des contre-célébrations de la conquête, il y a des raisons à cela. C'est, en effet, l'Indien pur de la fiction occidentale ou le héros de l'Europe biblique expansioniste qu'on s'efforce de célébrer, pendant que le franco invisible et ubiquiste ne demeure qu'un personnage de l'entre-deux, éternel métis dont on ne sait - et dont on n'a jamais su - que faire.

Depuis Pontiac jusqu'à Kérouak, en passant par Toussaint Louverture et Louis Riel, les plus grands personnages de la Franco-Amérique - héros transcendants d'une géographie composite, entre la mouvance et le marronage, la trail et la route - auront sans cesse servi de levain aux autres Amériques. Née de sa propre disparition, la Franco-Amérique aura toujours été une aventure de l'tn-between. Ainsi, aura-t-elle eu beau incarner, depuis Haït jusqu'à l'Athabaskie, et de l'Isle-à-la-Crosse jusqu'à l'Isle-à-la-Tortue, la plus grande célébration occultée de la Renaissance, la fête ou l'anti-fête est ailleurs.

Durant toute la période coloniale française, il y aura toujours eu, en fait, une multitude d'Amériques se chevauchant sans trop le savoir: l'Amérique autochtone, l'Amérique des Français, l'Amérique esclavagiste, et une quatrième instance, majoritaire, ambivalente et imprécise, procédant des unes et des autres. On nous a appelés et on continue de nous appeler French, mais nous savons très bien que nous ne sommes pas et n'avons jamais été ni Français, ni Français d'Amérique, mais bien autre chose: des Métis sans appellation précise et portant de ce fait tous les noms. Bref, une espèce d'Amérique sans nom ayant donné son âme à l'Amérique entière.

N'ayant jamais fait son indépendance contre l'Europe ailleurs qu'à Saint-Domingue (et dont Haïti continue de payer fort cher le prix jusqu'à ce jour), la Franco-Amérique n'a jamais accompli la révolte fondatrice que connaîtront tous les autres pays du Nouveau Monde. Ce qui plus est, alors que nous allions constituer, tout au cours des XVIIlème et XIX ème siècles, l'une des expériences 
géographiques qui aura forcé l'admiration de tous les chroniqueurs et les voyageurs, pourquoi faut-il qu'on se retrouve, en cette fin de vingtième siècle, en manque chronique d'identité. Que s'est-il donc passé pour qu'une aventure auréolée d'une telle gloire chez les auteurs anglo-saxons, arrive aussi mal à nous inspirer collectivement?

Pourquoi avoir maintenu, à travers les manuels scolaires et l'histoire officielle, ce qu'il faut bien appeler une conspiration du silence autour de la géographie franco-amérique? D'où vient une telle honte au moment où les Francis Parkman et bon nombres d'écrivains, depuis Longfellow et Fenimore Cooper jusqu'à James A. Michener et Brian Moore. allaient puiser dans notre réalité les héros et les hèroïnes que nous refusions d'y déceler?

Parallèlement, les Français qui ont perdu l'Amérique aiment bien se consoler en se répétant:

Voici 468 ans [500 maintenant] que le nom d'Amérique a été prononcé et écrit pour la première fois. Et ce fut en France.

Mais, au fait, que s'est-il passé? Voltaire a fait célébrer par une messe la conquête du Canada; Napoléon a vendu la Louisiane; Choiseul a échoué dans son projet d'un pays franco en Amérique du Sud tout comme Maximilien au Mexique. Et, lorsque Pontiac au large des Appalaches, Toussaint Louverture à Saint-Domingue, Louis Riel dans le Nord-Ouest, lorsque tous ces libérateurs ont fait appel à leurs frères afin de réaliser, bien avant Bolivar, le rêve d'une grande Amérique, pourquoi avons-nous refusé de leur tendre la main d'un bout à l'autre d'un continent que nous avions parcouru sans relâche? Beaucoup de Francos sont morts non pas de la main des Anglais mais par désaffection de leurs propres compatriotes. Tant que la Franco-Amérique n'aura pas assumé un tel héritage, pour mieux le dépasser, la question identitaire perdurera.

On te déclare que tu es québécois, que d'autres sont Fransaskois, Martiniquais, Louisianais, Cajuns, Canucks, Haïtiens, Franco-ténois, French-cree, etc. Mais pourquoi au juste un si grand nombre de tribus sans que jamais ne se soit présenté 
un seul grand chef - un seul Sachem - ayant su faire miroiter, à travers l'espace et au-delà du temps, un destin commun à partir d'une origine commune?

Peut-être ny a-t-il vraiment jamais eu une origine commune pour rassembler sous une même bannière, tout au moins symboliquement, cette Amérique-in-themaking. Malgré tous les efforts des historiens officiels pour définir un pure breed Canadien-français au-delà de tout soupçon, nous avons toujours procédé, depuis les touts débuts, d'une origine mouvante. Comme les tribus qui nous ont reçus sous leurs wigwams et leurs tee-pee, nous portons les noms des géographies que nous avons empruntées et les prénoms des rivières que nous avons parcourues. Fleuves et cours d'eau qui sont devenus, les uns après les autres, le patrimoine de l'Amérique entière, sauf le nôtre!

Nés précisément de toutes les Amériques qu'on aura voulu inventer à même notre trajectoire géographique, c'est pourquoi nous demeurons le peuple hors célêbration de tous les quincentenaires... .

Nous ne pouvions certainement pas découvrir le nouveau monde puisque nous sommes le nouveau monde.

Évidemment, on a toujours peur de se l'avouer, mais un minoritaire aura effectivement trois langues. Une langue maternelle toujours à moitié perdue, une deuxième langue à moitié inventée, et une troisième à cheval entre les deux. Sauf qu'il y a toujours le risque que le cheval s'affole ou prenne le mors aux dents.

Si bien que le Franco n'est vraiment bien qu'entre deux chaises, là où tous les autres ont mal. Car ce lieu inconfortable finit toujours par se transformer en espace de création idéal? Ce n'est ni en Virginie, ni en Californie que le jazz est né, mais en Nouvelle-Orléans après l'arrivée des Créoles haïtiens qui fuyaient Saint-Domingue. Alors... .

C'est faute d'avoir inventé, dira-t-on, la caravelle, la nacelle, ou la sarcelle à quatre ailes qui auraient pu servir de désignation au projet Franco-Amérique, que nous n'avons jamais pris conscience de notre force. La grandeur de l'Amérique française, les vestiges de l'Amérique française, le je-ne-sais-quoi de la French America, etc., etc. Tous ces noms relèvent d'un vocabulaire tellement éculê, et s'ils continuent à être utilisés, c'est justement parce que la France a perdu son Amérique qui aura toujours été moins la sienne que la nôtre. 
Il y a quelque chose de pathétique dans cette recherche désespérêe de l'Amérique sans nom qui nous a donné naissance. Si nous avions réussi à inventer une Amérique canadienne, une Amérique louisianaise ou une Amérique haïtienne, sous lesquelles regrouper nos effectifs incertains, nous serions tous Canadiens, Louisianais ou Haïtiens, et pourrions le lancer à la face du monde. Take it or leave it, but it doesn't matter. I know who I am. I am what I know. I am what I am. But do I am what I know?

Je ne crois pourtant pas qu'il y ait, du moins pas pour le moment, la naissance d'une d'Amérique québécoise, parce que l'identité Québec a été paradoxalement fondée sur l'établissement de nouveaux liens avec l'Europe. Le refus d'Amérique et le refus du métissage qui s'ensuivent corollairement ne sauraient donc servir de lieu de rencontre à des peuples que le Québec a rejetés d'emblée dans son propre projet identitaire. Au moment précis où le Québec, en tant qu'instance résiduelle de la Franco-Amérique, prétend faire sa souveraineté, la récente fermeture du Secrétariat permanent des Peuples francophones, à Québec, en révèle long sur tout cela. $^{2}$

Quand je pense à tous les noms d'emprunt qu'on s'est donnés pour mieux se cacher le fait que nous n'avons pas su ni même essayé d'imaginer un nom commun à tous. Mais pourquoi donc?

J'ai constamment employé, pour ma part, l'expression Franco-Amérique pour tenter de renverser le courant européocentrique que véhicule forcément l'idée d'Amérique française. Et aussi, pour faire avaler, dans une grande geste anthropophage, le franco par l'Amérique et projeter ainsi une désignation qui ne soit pas d'outre-atlantique, mais je ne suis pas dupe et je sais que ça ne va pas tout à fait.

Je suis trop conscient que quelque chose manque, de façon peut-être tragique. Faute d'avoir jamais trouvé l'appellation susceptible de faire émerger une destinée commune, nous avons raté notre diaspora, du moins jusqu'à maintenant.

Des amis "Noirs" de la caraỉbe m'ont souvent avoué, textuellement, ce qui suit. "Si vous n'aviez pas été "Blancs," vous les Québécois, mais bruns, marrons ou gris, il y aurait eu un signe distinctif auquel vous reconnaître vous-mêmes en deçà et au-delà de toute origine et de toute assimilation. Dans une telle optique, vous auriez alors réussi à imposer cette reconnaissance aux autres, comme ce fut le cas du 
black power et prêsentement du poder chicano-latino. Mais vous n'avez pas cela. Votre drame, c'est de vous être perdus dans la masse anglo sans possibilité de rémission et d'identification spontanée par les autres."

Je pense qu'ils ont passablement raison; mais je me refuse de le croire tout à fait. Je sais bien qu'il y a des "Noirs" qui employent des "détergents" et autres lotions pour se blanchir tandis que nous, nous employons des dictionnaires et des "offices de la langue française" pour blanchir notre langue. Cela revient au même, n'estce-pas?

Quand on a comme critère distinctif, plutôt que la couleur de sa peau, la couleur d'une langue - chiaque, cajun, joual ou créole - que tous combattent avec violence, à commencer par la France et tous les Anglos dont le modèle demeure la France, il n'y a guère d'autre choix que de faire de la musique. C'est ce qu'un André Gladu a fort bien compris en allant chercher dans son cinéma le Son des Français d'Amérique. ${ }^{3}$ Il a réussi là quelque chose d'inouï: faire surgir des tréfonds de l'Amérique, le reel-jazz transcendant des ruine-babines du Nouveau-Monde.

Vive l'Amérique sans nom!

I'm not speak French anymore, baby, but I do play it.

Il y a toujours eu pourtant des Canadiens pour dire que l'Amérique ne s'était pas faite en français. Ce n'est pas qu'ils ne connaissaient pas leur langue, c'est tout simplement qu'ils ne connaissent pas ou n'ont pas voulu connaitre l'Amérique.

Il était une fois une vieille Indienne de la Saskatchéouanne qui entreprit un jour de raconter sa vie en commençant par ces mots: "Oh! vous savez, en ce temps-là, il n'y avait que des Indiens et des Canayens, les Blancs sont arrivés bien après." Si un tel témoignage se passe de commentaire, il renvoye à une réalité sur laquelle on ne saurait trop insister.

"The designation of French is often indifferently applied to Canadians [that is Canadiens], Métis of all grades, and even pure Indians who associate with Métis and speak their patois. In a general way it may be asserted that, north of the fortieth parallel, from Quebec to Vancouver Island, there is scarcely a native tribe, from the Sioux to the Esquimo, that has not been tinctured with French blood." ${ }^{4}$ 
Si les autochtones se retrouvent donc tous un peu frenchés sur cette terre de liberté appelée Amérique, quel avenir était-il réservé aux uns et aux autres, au terme d'un mélange aussi prometteur?

"Ils disparaitront comme une vapeur de la surface de la terre; leur histoire se perdra dans l'oubli et les lieux qui les connaissent encore en perdront pour toujours le souvenir., ${ }^{5}$

"Voilà tout ce qui reste d'une nation jadis puissante; on peut dire que, prochainement, on verra disparaître ses derniers représentants, mais, si un destin aussi triste est inévitablement réservé à cette race, on peut ajouter cependant qu'elle fait une belle mort. ${ }^{6}$

"Encore un peu de temps et l'homme blanc aura cessé de nous persécuter, car nous aurons cessé d'être, confiait un aïeul au dernier quartier de lune."

Qu'est-ce à dire? Le message-génocide des Blancs paternalistes est allé si loin que les ressortissants eux-mêmes en sont venus à accréditer leur propre disparition comme pour mieux respecter une histoire dont on les avait bannis.

\footnotetext{
"Un Canadien errant banni de ses foyers

parcourait en rêvant des pays étrangers

Un jour, triste et pensif, assis aux bords des flots.

au courant fugitif, il adressa ces mots:

si tu vois mon pays, mon pays malheureux

va dire à mes amis que je me souviens d'eux.
}

Personne, pas même la géographie, n'aura bientôt su si le pays avait existé ou pas!

En guise de pied de nez à toute assimilation, et pour faire émerger la géographie sous-jacente à l'Amérique anglo, je m'en voudrais de ne pas rapporter ici à loisir un extrait de lettre du Métis Gabriel Dumont, que mon collègue Pierre Anctil a sortie des décombres de l'histoire pour la présenter, non sans émotion, en ces termes. "Il se dégage une singulière poésise Ide la narration que donne l'ex-chef métis de son dernier voyage dans le Nord-Ouest] de tant de noms aujourd'hui anglicisés: Yellowstone River, Sun River. St. Peter's Mission ... étrange itinéraire surgi tout d'une venue, parcouru, on dirait, à bride abattue tant les toponymes se succèdent rapidement $[\ldots]$ : 
Je vous raconterais tout au long mon voyage lorsque j'aurais le plaisir de vous voir, je vous citerais seulement ici, les places où j'ai passé; en partant d'ici - je suis passé à Lewiston, Montana, où j'al rencontré les prémiers Métis, de là j'ai été à la Dépouille et de là voir les Piedgoms à la rivière aux Boullets après les avoir vus j'ai filè le long de la Montagne comme en revenant d'Orcha, les bords de la rivière au Soleil jusqu'à la Mission de St-Pierre, de là je suis revenu à Lewiston, Montana; j'ai mis deux mois à faire ce voyage pour revenir à Lewiston, de là je suis passé à Claquette dans le Missouri et de là au fort Assinniboine sur la frontière américaine, de là j'ai filé le long de la rivière au Lait jusqu'à l'entrée du Missouri, c'est-à-dire où la rivière au Lait entre dans le Missouri jusqu'aux réserves indiens, que j'ai suivis, d'abord les Sioux et ensuite les Assenneboins le long de la frontière; j'ai omis de vous nommer le Fort à la rivière aux Trembles et de la Roche Jaune, je suis revenu dans le Dakota, à la Montagne aux Tortues et à Saint-Jean où il y a davantage de Métis, là se trouvent les réserves de Métis et de Sautteux après la Montagne aux Tortues, je suis arrivé à Olga, Cavalier County, Dakota, de là à Neche Dakota oủ j'ai pris les Chars pour m'en revenir à New-York. ${ }^{7}$

"A elle seule, commente Anctil, cette lettre fait basculer l'univers des Métis; elle rompt la conspiration du silence qui les entourait à l'époque aux États-Unis." Il est donc un fait qui saute aux yeux. Cette Amérique mi-indienne mi-franco qui devait toujours mourir inexorablement est en train de revivre d'un bout à l'autre du continent, depuis l'Alaska jusqu'à la Patagonie.

Alors que cette fin de siècle se fait un lieu de renaissance, on se prend à rêver. Et si notre dépossession, par un jeu de reconquête imprévu, n'allait pas se transmuter en triomphe pour jeter les bases d'une identité aussi imprévue que nouvelle, susceptible de renouveler l'Amérique de l'an 2000! J'écris ces lignes sans restriction. Et que tous les pourfendeurs d'utopie, qui n'ont jamais manqué loccasion de décocher leurs dards, soient renversés par leur propre défaitisme.

Nous sommes, avec et après les autochtones, la dernière Amérique à ne pas avoir accompli sa souveraineté au siècle passé, nous serons la première à marquer le tournant du siècle. 
Que conclure?

Combien de cheveux bouclés, emportés par-dessus le bastingage de l'histoire, sont allés chercher, sous d'autres cieux et chez d'autres manitous, l'inspiration et les matous que le pays ne pouvait produire! Et pourquoi pas?

Lorsqu'on parcourt la carte de la Nord-Amérique, on y rencontre soudain, du côté du XVII ème siècle et du Missouri, un arbre sur lequel sont gravées des fleurs-de-lys ainsi que les armes de la France. J'ai retrouvé cet arbre, apparemment mort depuis des décennies. Des gens en uniforme s'étaient amusés à lui tirer dessus, mais son écorce vivait toujours. S'il est difficile de faire pousser des tiges à partir de l'écorce d'un arbre décédé, eh bien, il purait que nous avons fait plus que cela. Nous avons construit un immense canot appelé rabaska pour inventer un monde nouveau en chantant:

c'est l'aviron qui nous mène, mène, mène, c'est l'aviron qui nous mène en haut!

Peuple non prẻvu à l'ordre du jour, ai-je dit! Mais pas du tout.

Peuple de l'en-haut jusqu'aux bayous les plus secrets du Mississippi et aux campements les plus imprêvus du Grand Nord.

Peuple de rapldes, de méandres de rivières dont on croyait avoir coupé le tronc mais qui est revenu par mille branches et mille affluents.

Voyez ce peuple qui coule lentement vers sa source depuis l'embouchure du Nouveau Monde.

Derrière la bannière des États-Unis et les irruptions volcaniques de la Carä̈be, sous les sédiments de la liberté, il existe une couche indélébile dont je suis le ressortissant triomphant.

C'est là que je suis né et c'est là que je vais perdurer. Quelles que soient les langues que je parle, que j'ai parlées ou que je cesserai de parler.

C'est là que je suis né entre le Saint-Laurent et le Mississippi, la rivière enfouie et la grande rivière, le Dè-tcho et l'Artibonite.

Alors aussi bien me l'avouer et m'en prévaloir une fois pour toutes. 
Après avoir été vendu par la France, vaincu et déporté par l'Angleterre, assimilé par les Yanquis, poursuivi par l'Espagne, par Saint Jean-Baptiste et par Dieu le père, mais défendu par le carnaval de la Nouvelle-Orléans, il est temps de revenir à la vérité du Mississippi.

Si certains peuples remontent vers leur origine; nous, Francos, descendons vers notre source!

Nous sommes la découverte que tous recherchent sans le savoir.

Au moment où la béance haïtienne renait ses cendres, au moment où le métis Léonard Pelletier, en prison depuis Wounded Knee revient hanter la conscience wasp, que reste-t-il d'autre que le Mississippi, que reste-t-il d'autre que notre réalité autochtone, mulâtre et créole, sinon un poème à double identité qu'on me permettra d'intituler, telle une goélette ou tel un bateau-pirate:

\title{
Le denise-jeannette ou la franco-amérique
}

\author{
elle est là she is there \\ seins en rêve \\ bras en sursis \\ elle est là
}

elle est there

à moitié assoupie à moitié submergée

un sourire flottant sur l'èchine

une paire de jeans sur ses pattes endormies

cheveux épars sur ses épaules brunes

mains couchées entre les cuisses

yeux en ovale

lèvres en lippe

she is là

son souffle murmure un son transparent

son ventre ondule sous l'embrun du temps

she is there elle est là

à moitié indienne à moitié frenchée

rien qu'une palpitation au détour de l'histoire. 
Jean Morisset 11

\section{Notes}

${ }^{1}$ Jacques-Donat Casanova in Une Amérique française (Paris/Québec: La Documentation française et l'Editeur officiel du Québec, 1975). La citation apparait en page 7.

${ }^{2}$ Durant plus de dix ans, le SPPF aura été le lieu où parvenait pratiquement l'ensemble des journaux et périodiques de la Franco-Amérique. Eh bien, comme on l'a fait pour les codexs aztèques et mayas, on a détruit tout ce matériel ayant jugé bon que le corpus franco de toute la nord-amérique ne valait pas la peine d'être conservé. Difficile de trouver plus "éloquent" exemple de ce que j'essaye de démontrer ici.

${ }^{3}$ Sous ce titre, c'est une série de 27 films (dont 4 sur la Louisiane) qui ont été co-produits et co-réalisés par André Gladu et Michel Brault avec la collaboration de la Société Radio-Canada. Le Son des Français d'Amérique est toujours disponible chez Multi-Média, à Montréal (5225, rue Berri, Qc H2J 2S4) et, incidemment, on en trouve une collection au Centre for Acadian \& Creole Folklore à l'Université de la Louisiane (South West Louisiana), à Lafayette.

${ }^{4}$ V. Havard (M.D., Assistant Surgeon, U.S.A.), "The French Half-Breeds of the Northwest" in Report of the Smithsonian Institution for the year 1879 (Washington, 1880). Les citations se retrouvent aux pages 314 et 317-18.

${ }^{5}$ Washington Irving "Des Sauvages de l'Amérique septentrionale" in Charles Waterton, Excursions dans l'Amérique méridionale, p. 465-466. La version originale s'intitule Wanderings in South America, the North-West of the United States and the Antilles in the Years 1812,1816,1820 \& 1824 With Original Instructions for the Perfect Preservation of Birds, etc. for Cabinets of Natural History (London: MacMillan \& Co., 1879), 520 p.

${ }^{6}$ Edgar Rochette, Notes sur la Côte Nord du Bas Saint-Laurent et le Labrador canadien (Québec: Imprimerie Le Soleil, 1926), p. 99.

${ }^{7}$ Lettre datée du 31 octobre 1887 et citée par Pierre Anctil dans "Les Lettres de Gabriel Dumont au Major Edmond Mallet» in Recherches amérindiennes au Québec, vol. X, Nos 1-2 (1980): p. 53-66. Citation à la p. 62.

${ }^{8}$ Voir Peter Matthiessen, In the Spirit of Crazy Horse (Viking Press, 1983 et 1991). 


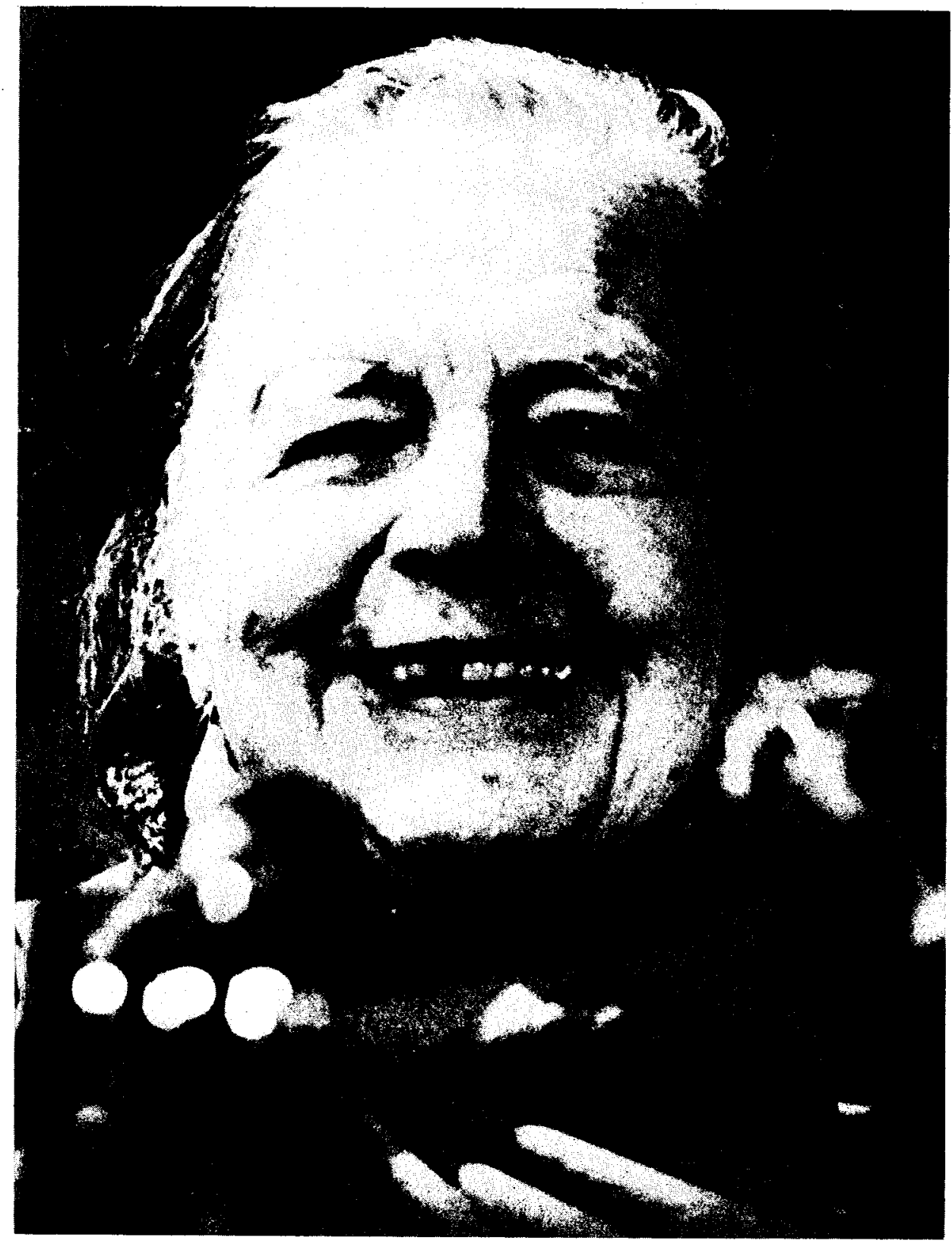

Marguerite Yourcenar. Photo: Louise Bilodeau Québec, le 30 septembre 1987 